\title{
High resolution measurements and modeling of auroral hydrogen emission line profiles
}

\author{
B. S. Lanchester ${ }^{1}$, M. Galand ${ }^{2}$, S. C. Robertson ${ }^{1}$, M. H. Rees ${ }^{1}$, D. Lummerzheim ${ }^{4}$, I. Furniss ${ }^{3}$, L. M. Peticolas ${ }^{5}$, \\ H. U. Frey ${ }^{5}$, J. Baumgardner ${ }^{2}$, and M. Mendillo ${ }^{2}$ \\ ${ }^{1}$ University of Southampton, UK \\ ${ }^{2}$ Center for Space Physics, Boston University, Boston, Massachusetts, USA \\ ${ }^{3}$ University College London, UK \\ ${ }^{4}$ Geophysical Institute, University of Alaska, Fairbanks, USA \\ ${ }^{5}$ Space Sciences Laboratory, University of California, Berkeley, CA, USA
}

Received: 3 September 2002 - Revised: 19 December 2002 - Accepted: 21 February 2003

\begin{abstract}
Measurements in the visible wavelength range at high spectral resolution $(1.3 \AA$ ) have been made at Longyearbyen, Svalbard (15.8 E,78.2 N) during an interval of intense proton precipitation. The shape and Doppler shift of hydrogen Balmer beta line profiles have been compared with model line profiles, using as input ion energy spectra from almost coincident passes of the FAST and DMSP spacecraft. The comparison shows that the simulation contains the important physical processes that produce the profiles, and confirms that measured changes in the shape and peak wavelength of the hydrogen profiles are the result of changing energy input. This combination of high resolution measurements with modeling provides a method of estimating the incoming energy and changes in flux of precipitating protons over Svalbard, for given energy and pitch-angle distributions. Whereas for electron precipitation, information on the incident particles is derived from brightness and brightness ratios which require at least two spectral windows, for proton precipitation the Doppler profile of resulting hydrogen emission is directly related to the energy and energy flux of the incident energetic protons and can be used to gather information about the source region. As well as the expected Doppler shift to shorter wavelengths, the measured profiles have a significant red-shifted component, the result of upward flowing emitting hydrogen atoms.
\end{abstract}

Key words. Ionosphere (auroral ionosphere; particle precipitation) - Magnetospheric physics (auroral phenomena)

\section{Introduction}

Renewed interest has recently developed in auroral hydrogen emissions, the spectroscopic signature of energetic proton precipitation into the atmosphere. While not usually the major source of particle energy flux associated with aurora

Correspondence to: B. S. Lanchester

(bsl@soton.ac.uk) (compared with electron precipitation) protons are an integral component of magnetospheric plasma that finds its way into the ionosphere. The proton component responds differently from the electron component to a variety of forcing and energisation mechanisms that eventually lead to precipitation into the atmosphere, producing auroral emission features.

Satellite and rocket-borne instruments yield in situ measurements of the energetic particle fluxes; global imaging provides the large-scale precipitation pattern. Hydrogen line profiles measured with ground-based instruments give information needed to study in detail the physical processes that protons undergo as they penetrate into the atmosphere. Since ground-based instruments make continuous observations at one location they are well suited to study the variability and temporal evolution of the proton aurora. To investigate the interaction of protons with the atmosphere requires good spectral resolution and appropriate time resolution. The instrumental resolution must, therefore, be sufficient to map accurately the line profile, in order to show the Doppler shift and broadening without masking any physical effects, such as a small wavelength shift of the peak. High spectral resolution also shows any contaminating spectral emission features in the profile.

We report the first results of measurements of the $\mathrm{H}_{\beta}$ line of hydrogen acquired at Svalbard (15.8 E, 78.2 N) with a new spectrograph, operating at a resolution (FWHM) of $1.3 \AA$ and with integration times as short as $10 \mathrm{~s}$. We show that these instrumental parameters are sufficient to identify clearly a redshifted wing of the profile and variations in the shape and peak of the emission profile. These measurements are well explained by a recently developed proton/hydrogen transport model (Galand et al., 1998). A short review of both measured and modeled hydrogen line profiles is presented in Sect. 1. It was fortuitous that several satellites measured the proton precipitation and resulting UV emissions over Svalbard during the proton aurora event measured from the ground. Particle data have been used as input to the Galand et al. (1998) model, providing the first direct comparison of hydrogen pro- 
files measured at high spectral resolution with model profiles using realistic energy spectra as input. The observations from the several coincident instruments are described in Sect. 2. The model is described briefly in Sect. 3 and in more detail in an Appendix. The results of the comparison of measured and modeled profiles are given in Sect. 4. The implications and future direction of this work are discussed in Sect. 5 and Sect. 6.

\subsection{Ground-based observations of auroral hydrogen lines}

The early history of auroral hydrogen emission line measurements has been well documented by Eather (1967). Vegard (1939) used a photographic spectrograph to make the first measurements of the $\mathrm{H}_{\alpha}$ and $\mathrm{H}_{\beta}$ lines from Oslo, Norway. He identified a diffuse $\mathrm{H}_{\beta}$ line that peaked $5 \AA$ shortward of the unshifted wavelength of a laboratory source. He later interpreted the observation to be due to neutralized protons entering the atmosphere at high velocity (Vegard, 1948). Gartlein (1951) confirmed the observation and interpretation with detection of the $\mathrm{H}_{\alpha}, \mathrm{H}_{\beta}$, and $\mathrm{H}_{\gamma}$ lines. With greater resolution Meinel (1951) obtained spectra that clearly showed that precipitating protons of very high velocity are responsible for an extended short wavelength tail in the line profile. Eather and Jacka (1966) made measurements in the magnetic zenith and the magnetic horizon directions, noting that the $\mathrm{H}_{\beta}$ line was broadened in both directions and that the zenith profile was shifted, in addition, toward shorter wavelengths. This observation provided definitive proof that energetic $\mathrm{H}$ atoms and the associated proton beam precipitating into the atmosphere are guided by the geomagnetic field.

Photographic recording of spectra required long exposure times, thus denying attempts at dynamic and morphological studies. Spectrophotometers were developed by Hunten (1955) and Montalbetti (1959) to increase the time resolution by using photoelectric detectors. Improvements followed the adoption of ever more efficient photomultipliers. The small slit area of spectrometers, however, is a basic limitation to the light gathering power of these instruments. The next step was the filter photometer developed by Omholt (1957). The large geometric factor, together with photoelectric detection, vastly improved the sensitivity (and time resolution) of the hydrogen line measurements. Initially, filters of $10 \AA$ FWHM were used which also had relatively poor rejection outside the design passband, allowing emission features other than hydrogen to pass into the detector. A channel measuring the background was required for a meaningful measurement. Zwick and Shepherd (1963) used a photoelectric Fabry-Perot (F-P) spectrophotometer with a filter to isolate a single F-P passband to obtain hydrogen line profiles and a background measurement. While this method yielded an unambiguous intensity measurement, the transmission was not as good as that of a filter photometer. Improvements in interference filters resulted in a narrower passband (a few $\AA$ ), a higher transmission, better rejection outside the passband, and a larger area, yielding excellent throughput. Continuous tilting of such a filter scanned the $\mathrm{H}_{\beta}$ line and the background radi- ation, allowing intensity measurements of the line as low as a few Rayleigh. This tilting-filter technique enabled Eather and Jacka (1966) to make much progress in studying auroral hydrogen emission.

Measurements of hydrogen line profiles at high resolution continued to prove challenging. Scanning spectrometers were favored for many years (Johansen and Omholt, 1963; Harang and Pettersen, 1967). The Fastie-Ebert design optimised throughput using long curved entrance and exit slits. Even so, long integration times are required to record the line profile with a minimum of noise. Since wavelength scanning records only a small segment of the line at a time, it is not practical to use a spectral resolution less than about $4 \AA$ for the weak hydrogen lines without resorting to long integration times. Fastie-Ebert scanning spectrophotometers were used to study hydrogen line profiles by Henriksen et al. (1985), Sigernes et al. (1994), Sigernes (1996), Lorentzen et al. (1998), Deehr et al. (1998), and Lummerzheim and Galand (2001). Application of CCD detectors to spectroscopy allowed for the simultaneous registration of all spectral elements of a line profile, resulting in a large sensitivity gain. Such imaging spectrographs are currently the optimal instruments for high spectral resolution measurements of auroral hydrogen lines (Baumgardner et al., 1993). One such instrument, the High Throughput Imaging Echelle Spectrograph (HiTIES), is described in Sect. 2.2 and used in the present study.

\subsection{Modelling of the hydrogen line profile}

Transport of fast protons into the atmosphere, their energy degradation and scattering, and their conversion to neutral atoms needs to be solved in the process of deriving Doppler profiles of radiating hydrogen atoms. The parameter required is the velocity distribution of the emitting atoms, equivalent to the wavelength dependence of the profile. Several theoretical models of proton/hydrogen transport have been developed and published, based on: a Monte Carlo method (Kozelov, 1993; Lorentzen et al., 1998; Synnes, 1998; Gérard et al., 2000; Solomon, 2001), a range theoretic method (Rees, 1982), or an explicit solution of the coupled proton/H atom transport equations (Basu et al., 1993; Strickland et al., 1993; Galand et al., 1998); but only a few include collisional angular redistribution, which is a key process to include in order to interpret the $\mathrm{H}_{\beta}$ profile at high spectral resolution.

Eather and Burrows (1966) adopted the formulation developed by Chamberlain (1961) to construct theoretical hydrogen line profiles for proton fluxes penetrating a dipole geomagnetic field with a range of energy and pitch-angle distributions. Comparison with observed profiles (Eather and Jacka, 1966) showed that the profiles cannot be modeled by assuming monoenergetic proton fluxes. However, either exponential or power law distributions and an isotropic pitch angle distribution yield a reasonable match to observed profiles. Söraas et al. (1994) used Chamberlain's derivation to 
construct synthetic line profiles that they compared with $\mathrm{H}_{\beta}$ observations from two sounding rockets.

Gérard et al. (2000) decribed the $\mathrm{H}^{+} / \mathrm{H}$ transport using a stochastic Monte Carlo approach. Their model includes collisional angular redistribution and was applied to the Lyman$\alpha$ Doppler profile. No comparison with observed Doppler profiles was proposed. Also using a Monte Carlo method (but without collisional angular redistribution), Lorentzen et al. (1998) compared their model output to the $\mathrm{H}_{\beta}$ Doppler profiles they acquired from Svalbard. The spectral resolution of the instrument was such that the red Doppler-shifted part of the profile was well within the instrumental broadening. The measured profile could be modeled without including collisional angular redistribution. Galand et al. (1998) solved the coupled kinetic transport equations including collisional angular redistribution, to model the $\mathrm{H}_{\beta}$ magnetic zenith Doppler profile. They were the first to show that when collisional angular redistribution is taken into account in the transport equations, a long wavelength tail is predicted, the signature of hydrogen atoms travelling out of the atmosphere (upward). Lummerzheim and Galand (2001) provided the first observational evidence for a red wing of physical origin using a $0.43 \mathrm{~nm}$ resolution spectrometer from Poker Flat, Alaska. They showed that the measured red wing was in agreement with that predicted by the model of Galand et al. (1998) through collisional angular redistribution.

The present work adopts the Galand et al. (1998) model with collisional angular redistribution to predict the $\mathrm{H}_{\beta}$ Doppler profile. The detailed shape depends on the precipitating proton energy spectrum and pitch-angle distribution.

\section{Observations}

\subsection{Proton event over Svalbard, 26 November 2000}

An unusually intense proton precipitation event occurred on 26 November 2000. It followed a sudden increase in the solar wind density from $10 \mathrm{~cm}^{-3}$ to $25 \mathrm{~cm}^{-3}$ and subsequent increases in solar wind velocity from $450 \mathrm{~km} / \mathrm{s}$ to $>600 \mathrm{~km} / \mathrm{s}$ measured by the ACE and Wind satellites. The main effect of this fast shock in the solar wind was observed on the ground at Longyearbyen at 12:00 UT, although bursts of hydrogen emissions were observed during the morning hours following initial, smaller increases in solar wind velocity and density. A short-lived negative excursion of the $B_{z}$ component of the IMF at around 15:00 UT resulted in a characteristic brightening of the emissions from proton precipitation in the cusp, which is the subject of a separate paper (Lockwood et al., 2003). The ground-based measurements that are described here were made at Longyearbyen (15.8 E, 78.2 N) on Svalbard, which was in the afternoon sector at the time of the largest variations in the optical emissions associated with the proton precipitation (Lanchester et al., 2003). The large scale effects of the proton event are seen clearly with data from the Spectrographic Imager (SI12)/FUV instrument on the Imager for Magnetopause-to-Aurora Global Explo- ration (IMAGE) satellite, which provides 5-10 s images of the Doppler-shifted Lyman- $\alpha$ emission at 2-min intervals of the entire auroral oval. This emission, like the $\mathrm{H}_{\beta}$ emission, is produced solely by proton precipitation into the atmosphere (Frey et al., 2001). Particle spectra from the FAST satellite and from the DMSP satellites F12 and F13 provide the input to modeling of the $\mathrm{H}_{\beta}$ profiles measured below the satellites, which passed very close to the Longyearbyen site.

\subsection{Ground-based Imaging Spectrograph HiTIES}

The High Throughput Imaging Echelle Spectrograph (HiTIES) provides simultaneous measurements at different noncontiguous wavelength regions (Chakrabarti et al., 2001; McWhirter et al., 2003; Lanchester et al., 2003). It employs an echelle grating used at high orders with a free spectral range of $33 \AA$. The data recorded to date have demonstrated that the imaging spectrograph is capable of measuring auroral emissions, in particular $\mathrm{H}_{\beta}$, with high spectral resolution and high time resolution. Measurements presented here are at $60 \mathrm{~s}$ integration, but measurements were made at $10 \mathrm{~s}$ resolution, which showed clear $\mathrm{H}_{\beta}$ profiles, albeit rather noisy. The field-of-view is a narrow slit of $8^{\circ}$ centred on the magnetic zenith. The data presented in this paper have been integrated over the central $4^{\circ}$ of the slit, which for an $\mathrm{H}_{\beta}$ emission has the effect of increasing the signal-to-noise ratio without any loss of information. Integrating data over a smaller angular range does not affect significantly the magnetic zenith profiles. However, the instrument has been designed to detect small spatial changes at high time resolution for dynamic auroral structures, during which the angular range of integration is kept as small as possible. In addition to the $\mathrm{H}_{\beta}$ spectral window, two others were used on this campaign to measure the $\mathrm{N}_{2}^{+} 1 \mathrm{~N}$ bands $(4635-4660 \AA)$ and (4690-4715 $\AA$ ) (Lanchester et al., 2003), which in the present work are used as an indicator of electron precipitation, useful for checking the background emission.

$\mathrm{H}_{\beta}$ emission profiles at $60 \mathrm{~s}$ resolution are plotted as a time sequence in Fig. 1a from 13:00-16:00 UT on 26 Nov 2000. The intensity scale is in arbitrary units (counts), which are proportional to brightness in Rayleighs. The position of the unshifted $\mathrm{H}_{\beta}$ line is marked at $4861.3 \AA$. This sequence shows that the intensity of the $\mathrm{H}_{\beta}$ emission increased in several bursts. The peak wavelength is shifted to shorter wavelengths throughout, the result of line-of-sight velocity of downward moving hydrogen. There is a significant red shifted wing in the line profile throughout the event, a consequence of upward moving hydrogen. The shift of the peak appears to decrease with time, which is seen more clearly in Fig. 2. Two profiles of the $\mathrm{H}_{\beta}$ line are shown from within the bursts in intensity at 13:33 UT and 15:12 UT (marked with arrows in Fig. 1a). The intensity scale is arbitrary, but subsequent figures use the same intensity axis (counts from 0 to 1000), in order for direct comparisons to be made. The instrument function is overlaid. The red shift and the peak shift are greater than the FWHM of the instrument function, and, therefore, cannot be explained by instrumental broad- 


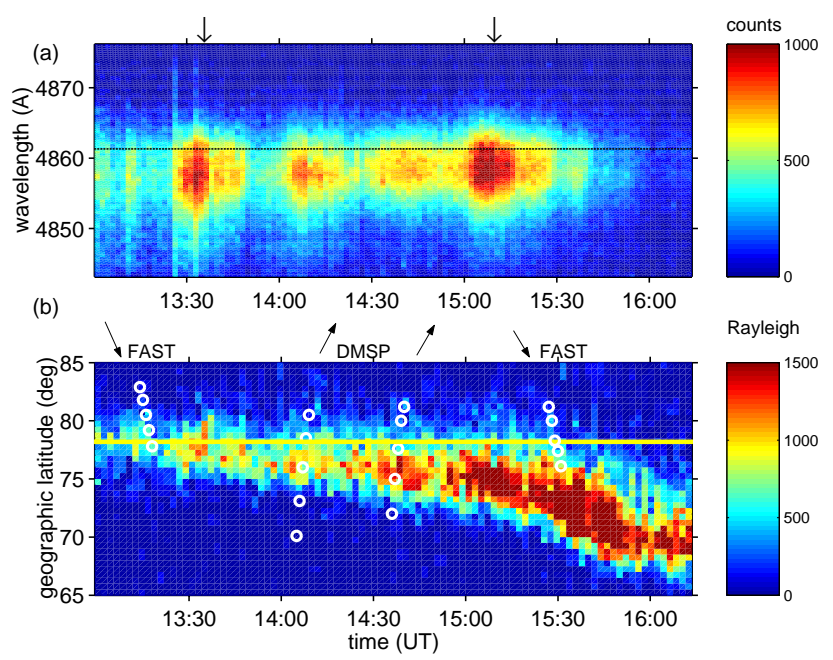

Fig. 1. (a) Time series of $\mathrm{H}_{\beta}$ profiles. The arrows mark the times of the profiles in Fig. 2. (b) Time history of Lyman- $\alpha$ intensity at longitude of Longyearbyen $\left(15.8^{\circ} \mathrm{E}\right)$. Times of the passage of FAST and DMSP satellites over the latitude of Longyearbyen $\left(78.2^{\circ} \mathrm{N}\right)$ are marked. Each pass has 5 points at one-minute intervals, centred on 13:16 UT, 14:07 UT, 14:38 UT and 15:28 UT, respectively.

ening. As will be shown later, the red wing is induced by collisional angular redistribution occurring inside the proton beam, confirming earlier observations by Lummerzheim and Galand (2001). Note that as well as a change in the shift of the peak, the profiles have also changed in shape (see Fig. 2). These profile shapes are the subject of this investigation.

\subsection{Measurements from above (IMAGE)}

Lyman- $\alpha$ images from the IMAGE/SI12 instrument have been combined in Fig. 1b, which is a time sequence of slices centred on the longitude of Longyearbyen $\left(15.8^{\circ}\right)$ and averaged over $1^{\circ}$ in longitude. The geographic latitude of Longyearbyen is marked at $78.2^{\circ}$. The hydrogen emission intensity measured from above at this latitude can be seen to vary with time in a similar way to that measured in $\mathrm{H}_{\beta}$ from below (Fig. 1a), with several distinct bursts. The equatorward drift with time seen in Fig. 1b has two components. The position of the oval moves to the south as the afternoon progresses, but superimposed on this is a very abrupt expansion of the oval following the southward turning of the IMF $B_{z}$ component. This expansion occurs at around 15:30 UT following a brightening of emissions in the cusp (Lockwood et al., 2003). This is then followed by a substorm in the 24 MLT sector, and a poleward return of the emission region. As a result of these events, the emissions measured over Longyearbyen near the start of the interval plotted in Fig. 1 are at the centre of a region of precipitation, but progressively they are from the northern edge of the optical features. However, there are certainly temporal changes within the large spatial movement southwards, also seen in the Meridian Scanning Photometer measurements of the $\mathrm{H}_{\beta}$

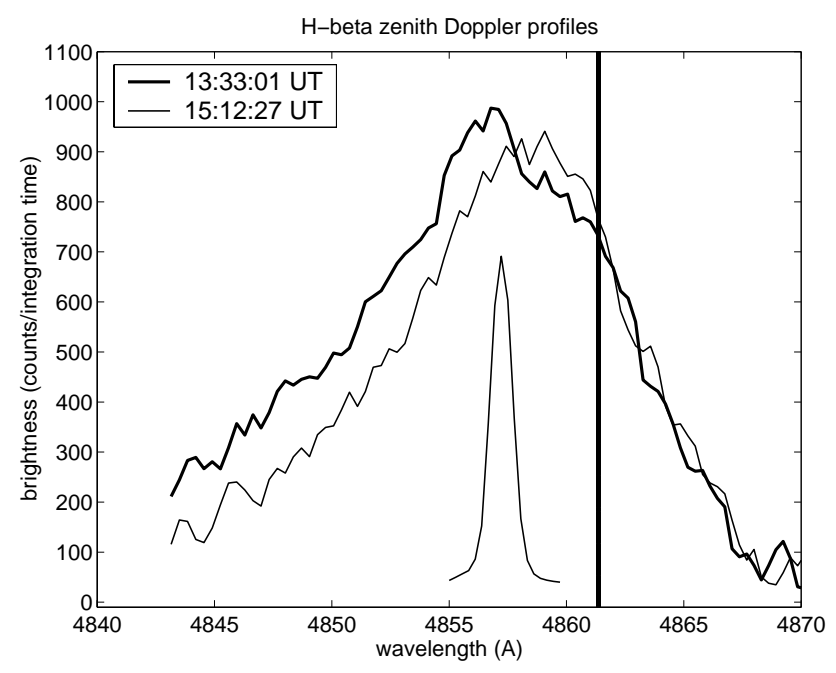

Fig. 2. $\mathrm{H}_{\beta}$ profiles at times of increased intensity (marked with arrows in Fig. 1a). The instrument function is superimposed.

emissions from Longyearbyen (not shown). Therefore, deconvolving temporal and spatial effects is difficult.

Plotted on Fig. 1b are the latitudinal coordinates of the FAST spacecraft for two consecutive passes around 13:16 UT and 15:28 UT, and of the F12 and F13 spacecraft at around 14:07 UT and 14:39 UT. The plotted points are one minute apart (see figure caption for exact times). It must be remembered that this is a temporal plot along the abscissa, and, therefore, the satellite positions marked here contain no longitude information. They act only to show the relative position in latitude of the satellites to the emission regions.

\subsection{In situ particle measurements (DMSP)}

Measurements from the pass of the F12 spacecraft closest to Longyearben are plotted in spectrogram form in Fig. 3. The satellite passes slightly to the east and north of Longyearbyen, which lies near the poleward edge of the region of proton precipitation at around 14:39 UT. The proton spectra from the F13 spacecraft when close to Longyearbyen at 14:07 UT are similar in energy and flux to the F12 pass in the region of proton precipitation, and are not shown here. There is no pitch-angle information as DMSP satellites view in the local zenith.

\subsection{In situ particle measurements (FAST)}

The two FAST passes during the interval under study show a temporal evolution between consecutive orbits, with an increase in the incoming energy flux measured by the satellite detectors. Mapping down the geomagnetic field to $500 \mathrm{~km}$ height is performed to provide spectra at the top of the ionosphere as input for modeling. This assumes there are no significant electric fields between the height of FAST and the ionosphere; no evidence for such fields is seen in either the electron or in the proton data. In the proton measurements used here, the fluxes are isotropic in the loss cone, which 


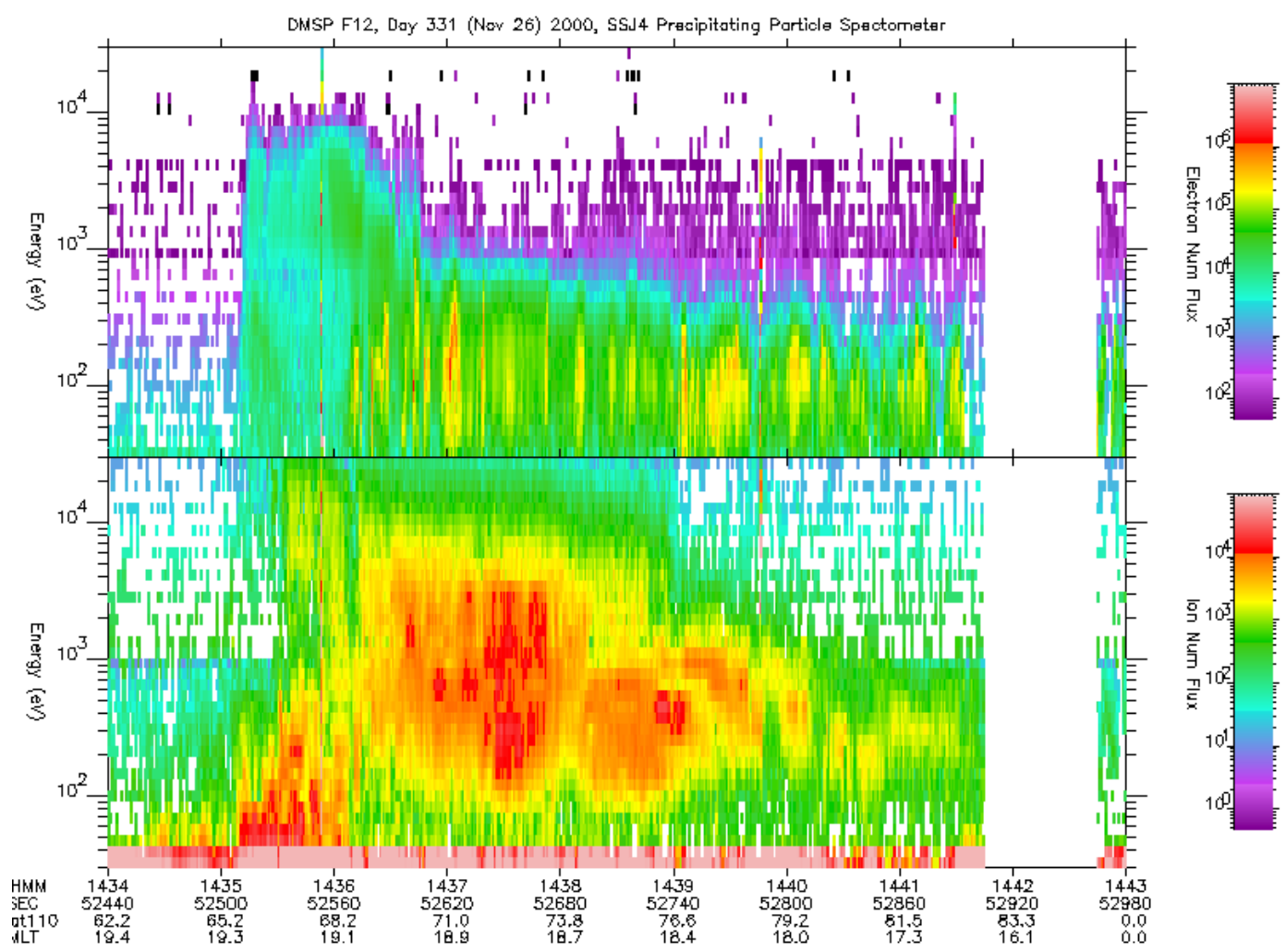

Fig. 3. DMSP F12 energy spectrogram of electrons (top) and ions (bottom).

is calculated to be $\theta<30^{\circ}$. Only protons within this loss cone will interact with the collision dominated ionosphere. Because of the isotropy, and the absence of a parallel electric field below FAST, the differential number flux of protons at FAST is equal to the differential number flux at the ionosphere.

For orbit 16927 shown in Fig. 4 the satellite passes to the east of Longyearbyen at 13:16 UT, but for the next orbit (16928) seen in Fig. 5 it is almost directly overhead at 15:28:30 UT. The top panel of these figures contains the energy flux carried by precipitating $\left(\theta<30^{\circ}\right)$ ions mapped to the ionosphere. This is a lower limit of the energy flux, as FAST measures only up to $28 \mathrm{keV}$, and from the lower panels it can be seen that ions of higher energies are likely to be significant. The second panel in each of Fig. 4 and Fig. 5 is the downgoing differential energy flux. The lower panels in these two figures show the full pitch-angle distribution, in order to emphasise the isotropy of the precipitation. Only spectra within the loss cone are used as input to the model. The loss of upgoing protons at $180^{\circ}$ pitch-angle is clear.

In the later pass, the spatial variation in the energy spectra as the satellite crosses the precipitation region shows a clear dispersion in energy, with energy decreasing with decreasing latitude. The position of Longyearbyen is within the separate region of precipitation poleward of the main region. It can be interpreted from the IMAGE data as structure along the polar cap boundary. Several discrete regions of emission are evident in the Lyman- $\alpha$ images.

\section{The Galand model of the hydrogen Doppler profile}

The model developed by Galand (1996) is a comprehensive proton transport code. It solves the steady-state Boltzmann equations for protons and $\mathrm{H}$ atoms and gives the proton and $\mathrm{H}$ atom fluxes as a function of altitude, energy, and pitchangle, starting from a specified incident flux at the top of the atmosphere. The solution includes the introduction of dissipative forces to simulate the energy loss from collisions, and includes angular redistribution from collisions and magnetic forces (Galand et al., 1998; Galand and Richmond, 2001). Collisional angular redistribution is taken into account through elastic collisions and through charge-changing collisions (i.e. capture and stripping). A phase function is included which can be considered as the probability function of angular redistribution through collisions. It has been successfully validated by comparison with rocket particle data of Söraas et al. (1974) and with the model of Basu et al. (1993) (Galand et al., 1997). Starting with an incident proton beam, the resulting $\mathrm{H}$ emission Doppler profiles are determined from the computed particle fluxes, neutral densities and emission cross sections. Since the transport equations are linear with respect to the incident proton flux, the $\mathrm{H}$ Doppler profile is directly proportional to the energy flux of the incident proton beam. A full description of the model is given in the Appendix. 

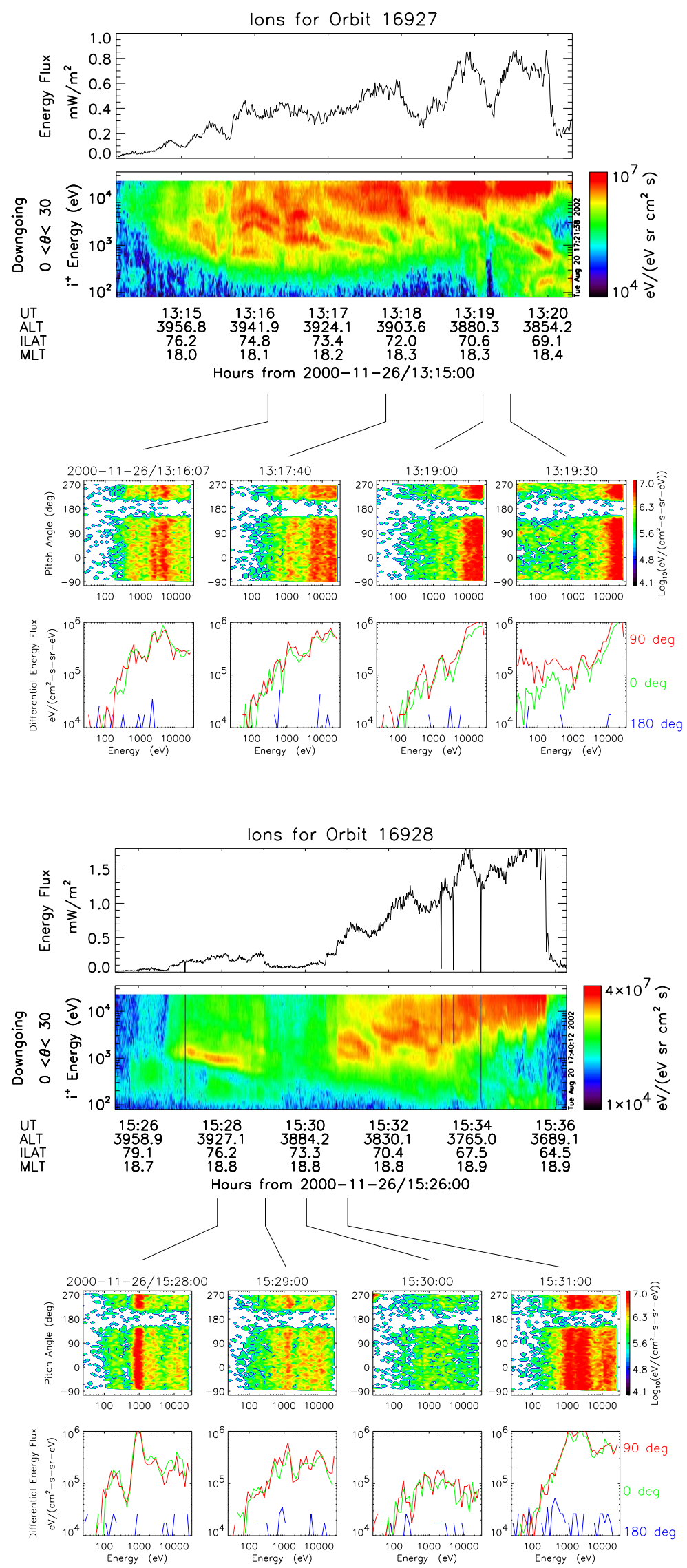

Fig. 4. (top panels) Energy flux of downgoing ions and energy spectrogram from FAST at around 13:17 UT. (bottom panels) Energy flux as a function of ion pitch-angle vs. energy, and as a function of energy for three different pitch-angles. The times are marked in the top panels.
Fig. 5. (top panels) Energy flux of downgoing ions and energy spectrogram from FAST at 15:28 UT. (bottom panels) Energy flux as a function of ion pitch-angle vs. energy, and as a function of energy for three different pitchangles. The times are marked in the top panels. 


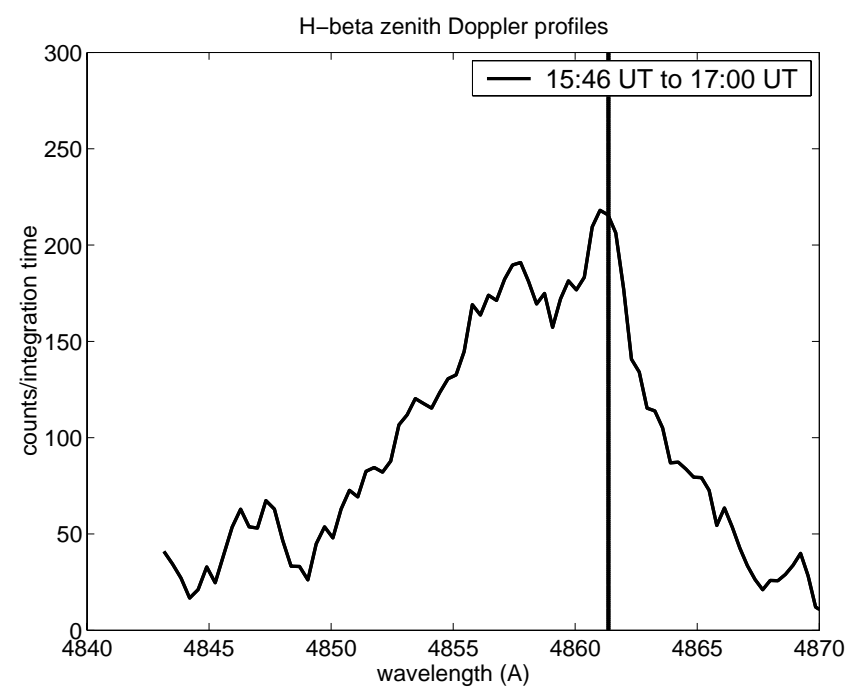

Fig. 6. Integrated $\mathrm{H}_{\beta}$ profiles showing geocoronal contamination when $\mathrm{H}_{\beta}$ emission is weak after 15:46 UT.

\section{Results: measured and modeled profiles}

\subsection{Analysis of the observed $\mathrm{H}_{\beta}$ profiles}

Flat fielding and dark current subtraction are applied routinely to the data frames from the HiTIES instrument. Wavelength calibration is performed using Fraunhofer absorption lines present in the scattered light solar spectrum measured during the twilight hours near noon at Svalbard. The uncertainty on the resulting wavelength scale is $\pm 0.05 \AA$.

Background subtraction is required to eliminate the effect of emissions from electron precipitation underlying the hydrogen emission. For the $\mathrm{H}_{\beta}$ profiles the region at the high wavelength end ( $>4870 \AA)$ of the data frame is used. Pixels beyond the red-shifted profile are averaged and subtracted from each frame. The effect of resonant scattered sunlight is a problem for the auroral $\mathrm{H}_{\beta}$ emission measurements between about 8:00 UT and 13:30 UT in late November. The spectrograph sees scattered solar $\mathrm{H}_{\beta}$ and other species in absorption throughout the "daylight" hours. When no cloud or precipitation is present, these data can be used in the wavelength calibration process. However, in the data presented below there was indeed proton precipitation from 12:00 UT onwards, and a method has been developed to extract the emission profiles resulting from proton precipitation from the sunlight contaminated background. The profiles from these times must always be treated with caution. In the results presented below the possible effect of sunlight will be discussed whenever it is relevant.

Other contaminating emissions are found to persist in the data. The most important is at the unshifted wavelength of the hydrogen line at $4861.3 \AA$. The likely cause is the resonant fluorescent component of geocoronal hydrogen emission, which varies in strength throughout the day and night, and appears to be strongest after bursts of proton precipita-

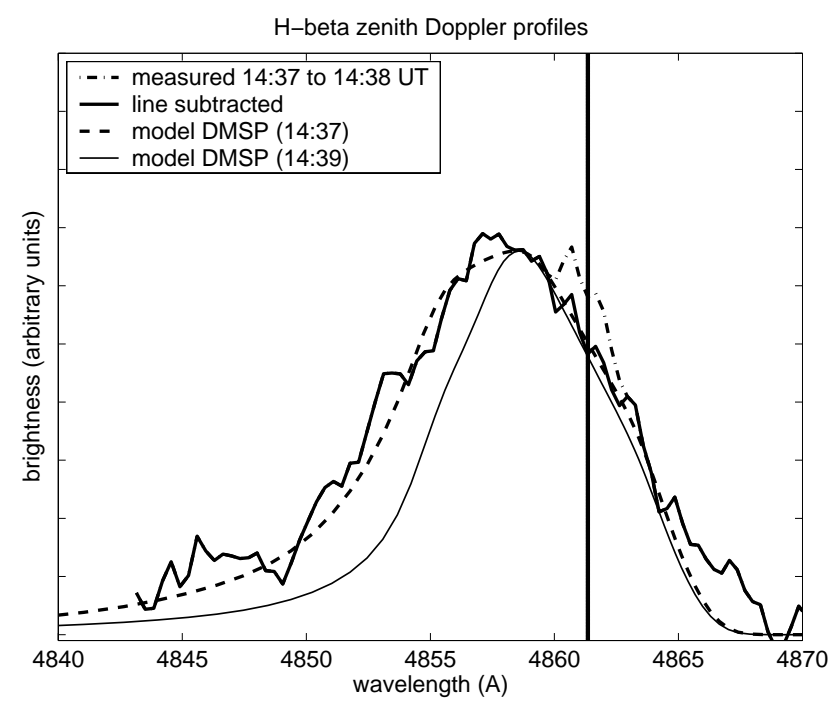

Fig. 7. Comparison of measured profile with modeled profiles using as input the spectra shown in Fig. 10. The dot-dash curve is the contribution of the unshifted line which has been removed in the solid curve.

tion. The occurrence of this emission is of interest in itself, and will be discussed elsewhere. It has been observed previously at auroral latitudes by Kerr and Hecht (1996), who used a Fabry-Perot spectrometer in quiet conditions to measure a weak ( 8 Rayleigh) emission of the $\mathrm{H}_{\alpha}$ line. In the present data the narrow unshifted emission line dominates the spectrum at times when the Doppler shifted emission is of low intensity. The effect of the unshifted line is shown in Fig. 6, which is an integration of all profiles between 15:45 UT and 17:00 UT. A Doppler shifted $\mathrm{H}_{\beta}$ profile is present, but it is weaker than the unshifted line at this time. In order to eliminate it from the Doppler profiles the unshifted line emission has been fitted to a Gaussian shaped profile, and then subtracted from the data. An example can be seen in Fig. 7 when the effect was clearly visible at the unshifted wavelength. The measured profile before the subtraction of the geocoronal line is plotted as a dot-dash line, and the resulting profile is the thick solid line. The other curves in this figure will be discussed below.

\subsection{FAST pass at 13:16 UT}

Sample ion energy spectra have been chosen from the satellite passes shown above (see Fig. 1b) to use as input to the model. The first FAST orbit at 13:16 UT does not make such a good conjunction with Longyearbyen as the subsequent orbit, passing to the east by several degrees of longitude. However, the two orbits indicate a temporal and/or spatial evolution of the proton precipitation at the latitude of Longyearbyen between 13:00 UT and 15:30 UT. The variability of the spectra at the time of the first pass is clear from Fig. 4, with a significant high energy component not measured by the spacecraft. The particle flux chosen as input to the model from 13:16:07 UT is plotted in Fig. 8. The parti- 


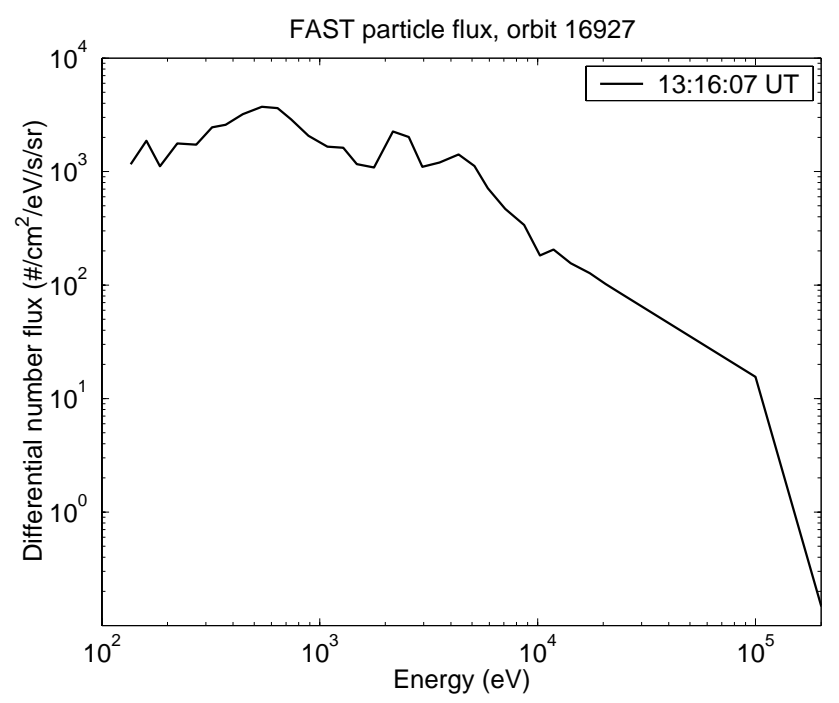

Fig. 8. FAST input particle flux at 13:16 UT with high energy extrapolation.

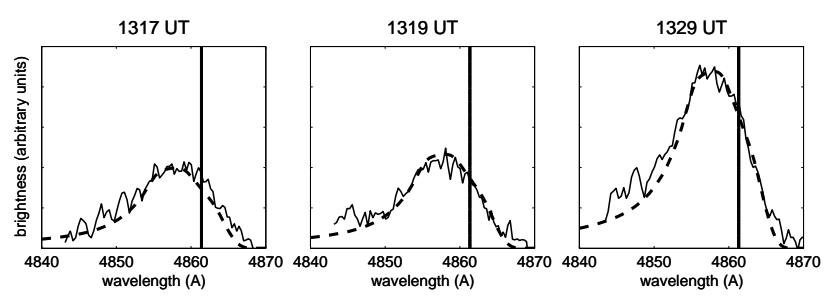

Fig. 9. Comparison of measured profiles at three different times with modeled profile, using as input the spectrum shown in Fig. 8.

cle data have been integrated over $3 \mathrm{~s}$, and mapped down the geomagnetic field to the ionosphere. Above $28 \mathrm{keV}$, where the measurements cut off, a high energy tail for the energy spectrum has been extrapolated for input to the model, as described in Sect. 2.5. Between $28 \mathrm{keV}$ and $100 \mathrm{keV}$, the last few energy bins of the measured flux are used for the extrapolation. At energies greater than $100 \mathrm{keV}$ the slope is taken from results of the Proton I rocket experiment (Söraas et al., 1974). This experiment was on the nightside, and, therefore, may not be the most appropriate shape. The high energy part of the spectrum is certainly an unknown in these data, particularly at times after 13:16 UT. It can be seen in the bottom panels of Fig. 4 that the measured energy spectra at 13:17:40 UT, 13:19:00 UT and 13:19:30 UT have peak energies much higher than $30 \mathrm{keV}$.

In order to compare the measured hydrogen line profiles with model results using this FAST pass, there are two problems: (1) the lack of good conjunction of the satellite with Longyearbyen and (2) the possible effect of solar absorption lines. The model profile is plotted in Fig. 9 and compared with three measured profiles (with solar absorption lines subtracted) during the interval of the FAST pass. These examples show the increase in intensity of the $\mathrm{H}_{\beta}$ emission over Longyearbyen between 13:16 UT and 13:29 UT. The model

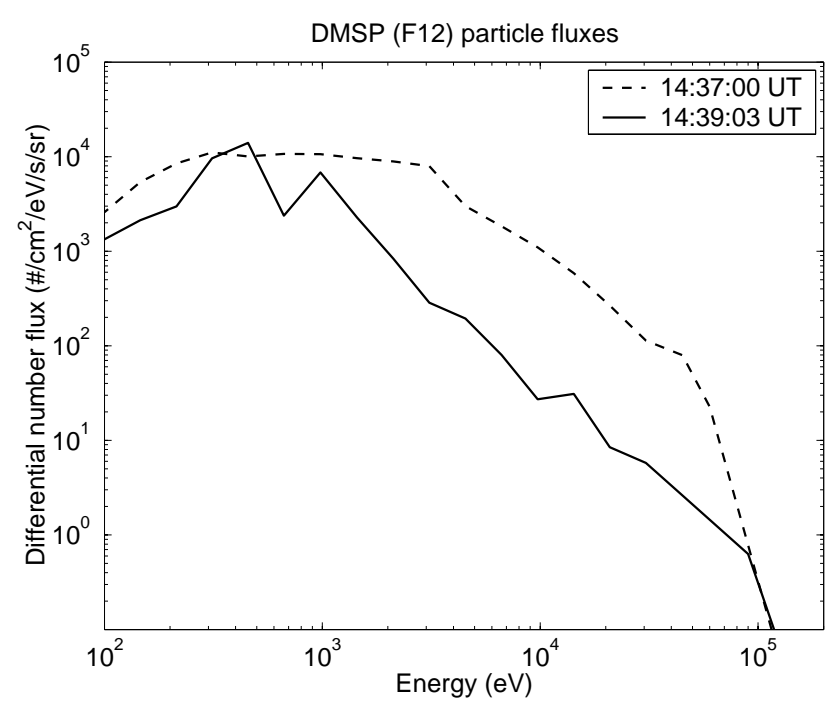

Fig. 10. DMSP F12 input particle flux at 14:37 UT and 14:39 UT with high energy extrapolation.

profile is scaled to the peak of each profile, as is the case in all subsequent figures comparing model and measured profiles. The overall fit is good, but there is a higher level of uncertainty for these data than with later examples, with a combination of solar absorption and geocoronal emission affecting the region around the unshifted wavelength of $\mathrm{H}_{\beta}$. However, we believe that the methods used to eliminate these effects are sufficient to provide excellent Doppler profiles during this time. The effect of the scattered sunlight was greater at the times shown in the first two panels, but was minimal by the third.

\subsection{DMSP pass at 14:39 UT}

The DMSP F12 satellite passed through the region of proton precipitation between 14:36 UT and 14:40 UT, with maximum energy flux measured between 14:37-38 UT when the satellite was to the east of Longyearbyen. It then passed to the north of Longyearbyen, by which time it was out of the main precipitation region. This can be determined from the Lyman- $\alpha$ emissions measured by IMAGE. For this reason, two spectra have been used as input to the model to show the difference between the middle of the precipitation region at 14:37 UT and a region at the northern edge at 14:39 UT. These are shown in Fig. 10 with extrapolation at large energies. The change from extrapolation to applied slope is made at $60 \mathrm{keV}$ for the 14:37 UT spectrum and at $90 \mathrm{keV}$ for the 14:39 UT spectrum, in order to make the most realistic high energy tail in each case.

Modeling of these input spectra show clearly that the mean energy of incoming protons has a large effect on the resulting profiles. The two model profiles are plotted in Fig. 7 with measured profile superimposed. As mentioned above, the contribution from the unshifted line is the dot-dash curve, and the measured profile after its subtraction is the thick 


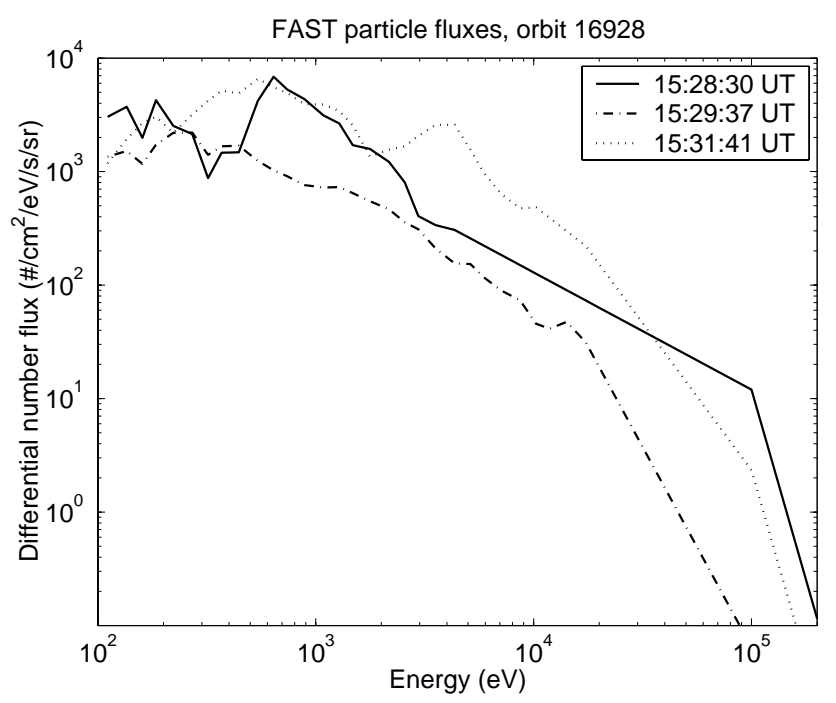

Fig. 11. FAST input particle flux with high energy extrapolation.

solid line (see Sect. 4.1). The model result using the input spectrum from the middle of the proton precipitation region makes a very good fit to the measured profile, whereas the input spectrum from the northern edge of the region underestimates the incoming proton flux at high energies significantly.

\subsection{FAST pass at 15:28 UT}

The closest approach to Longyearbyen was by the FAST satellite between 15:28 UT and 15:29 UT. The distributions in pitch-angle and energy can be seen in the lower two sets of panels in Fig. 5 at one-minute intervals between 15:28 UT and 15:31 UT. The downgoing flux of ions was isotropic at this time. The bottom panels of Fig. 5 show how the ion energy spectral shape varied. There is a clear, almost monoenergetic peak of $1 \mathrm{keV}$ at 15:28 UT, which decreases gradually in energy with decreasing latitude (as seen in the second panel of Fig. 5). By 15:29 UT this distribution has reduced in intensity, forming a gap in precipitation as the satellite travelled equatorward, entering the region of more intense and higher energy proton precipitation by $15: 31$ UT.

Spectra from three chosen times $(15: 28: 30$ UT, 15:29:37 UT and 15:31:41 UT) have been averaged over $3 \mathrm{~s}$, and mapped down the geomagnetic field to the ionosphere (Fig. 11). They have had the same extrapolation procedure at high energies as the spectrum shown in Fig. 8.

The model results for these spectra are plotted in Fig. 12. The short wavelength wing decreases with decreasing mean energy of the incident proton flux, which is $14.0 \mathrm{keV}, 4.9 \mathrm{keV}$ and $7.7 \mathrm{keV}$ for the 15:28 UT, 15:29 UT and 15:31 UT spectra, respectively. The measured profile of $60 \mathrm{~s}$ integration at this coincident time is superimposed (thick line). The integration interval is from 15:27:36 UT to 15:28:36 UT, which covers the time of closest approach of the FAST satellite to Longyearbyen. There is very good agreement over the whole

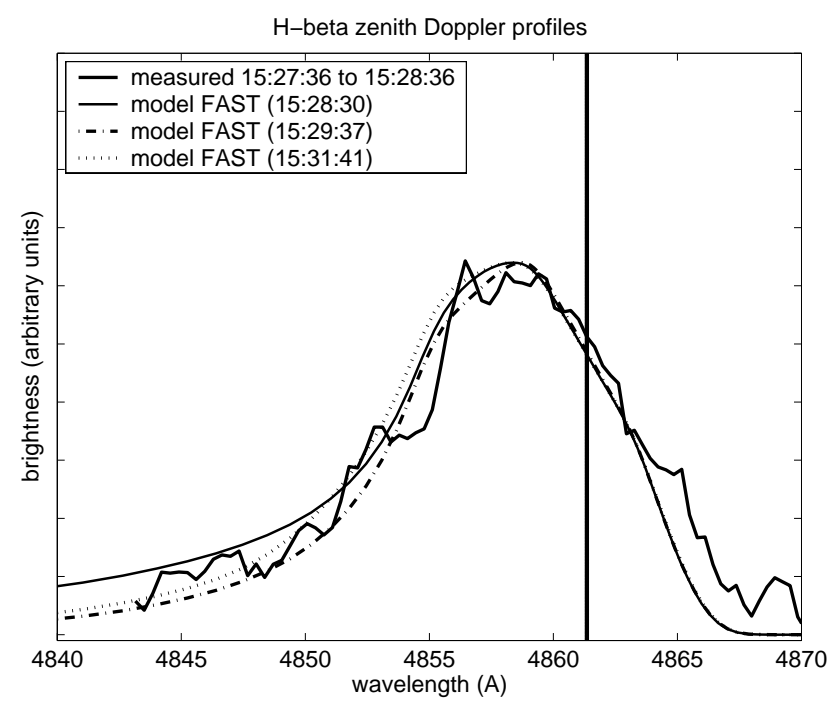

Fig. 12. Comparison of measured profile with modeled profiles using as input the spectra from FAST at 15:28:30 UT, 15:29:37 UT and 15:31:41 UT shown in Fig. 11.

profile, apart from the extreme red wing. Although the blue wing is sensitive to the extrapolation of the incident proton flux at high energies, changing this input results in no change to the red wing of the model profile, which is the result of upward-moving hydrogen. Angular redistribution is most effective at low energies. These upward moving hydrogen atoms result from the precipitating protons after they have undergone energy degradation in the atmosphere. The shape of the red wing is thus almost independent of the energy distribution of the precipitating protons at the top of the atmosphere.

In order to give a smoother profile for comparison with model profiles, an integration over the time of the increased flux (15:05 UT to 15:31 UT) is shown in Fig. 13, along with the same model profiles as shown in Fig. 12. Apart from the far red and blue wings, the profile fits the shape of the model results very well. It is possible that other contaminating emissions are present (e.g. see Fig. 6) at either side of the $\mathrm{H}_{\beta}$ Doppler profile, at around $4847 \AA$ and $4869 \AA$. No attempt has been made to remove these effects from the present data, and the physical processes creating these increased emissions have not yet been identified.

\subsection{Profile fitting}

From Fig. 1 and Fig. 2 it appears that there is a change in the wavelength of the peak intensity through the afternoon, from shorter to longer wavelengths. This would result from a decrease in the mean energy of the incoming protons, or from a change in the energy distribution. The FAST ion data indicate that the mean energy was indeed higher at the time of the first pass (13:16 UT). The energy flux was greater at the time of the later pass (15:28 UT). This is also confirmed by the HiTIES measurements, which registered the greatest 


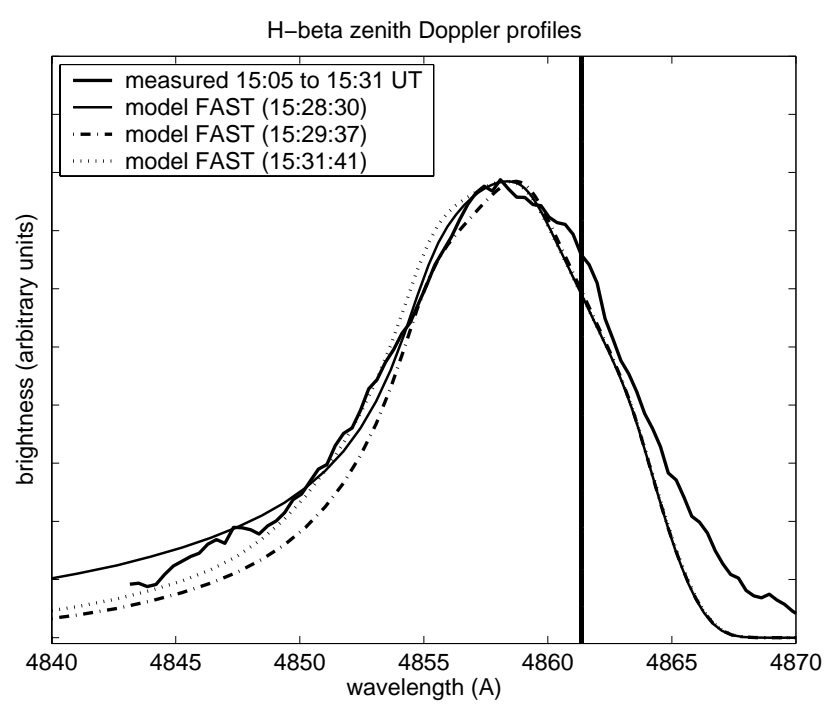

Fig. 13. Integrated $\mathrm{H}_{\beta}$ profiles at time of increased intensity between 15:05 UT and 15:31 UT. The same model profiles shown in Fig. 12 are included.

intensities between 15:00 UT and 15:30 UT.

In order to investigate the possible change in the peak wavelength, the measured profiles have been analysed using a method described in Lummerzheim and Galand (2001). A synthetic line profile is constructed from a Gaussian shape with different half widths on the blue and red sides of the peak, thus accounting for the different Doppler shift produced by precipitation towards the instrument and scattering of hydrogen away from the instrument, respectively. These synthetic spectra are fitted to the measured profiles, using a nonlinear least-squares fitting method. This method has been used to give a summary of the changes in the shape and peak intensity of the measured profiles. The results are shown in Fig. 14. There is evidence of a small variation of the peak wavelength $(\sim 1.5 \AA)$ between 13:50 UT and 15:29 UT. There is also a steady reduction of the width of the blue wing between 13:00 UT and 14:30 UT. It then remains constant. The red wing (not shown) does not change appreciably after 14:00 UT, but is wider and more variable in the earlier profiles. The data after 16:00 UT have a low signal-to-noise ratio. It is also apparent from the spread of the fit parameters that the most reliable estimates are from the periods of increased emission (see bottom panel of Fig. 14). Figure 15 shows the fitted and measured profiles for four selected times with the peak wavelength marked. This figure shows that even when the intensity is at a lower level between the bursts of emission, the fitting procedure is reasonable.

\section{Discussion}

The events of 26 November 2000 proved to have many interesting features, some of which have been reported elsewhere (Lockwood et al., 2003). These events provided the first clear measurements of $\mathrm{H}_{\beta}$ profiles made with the HiTIES instru-
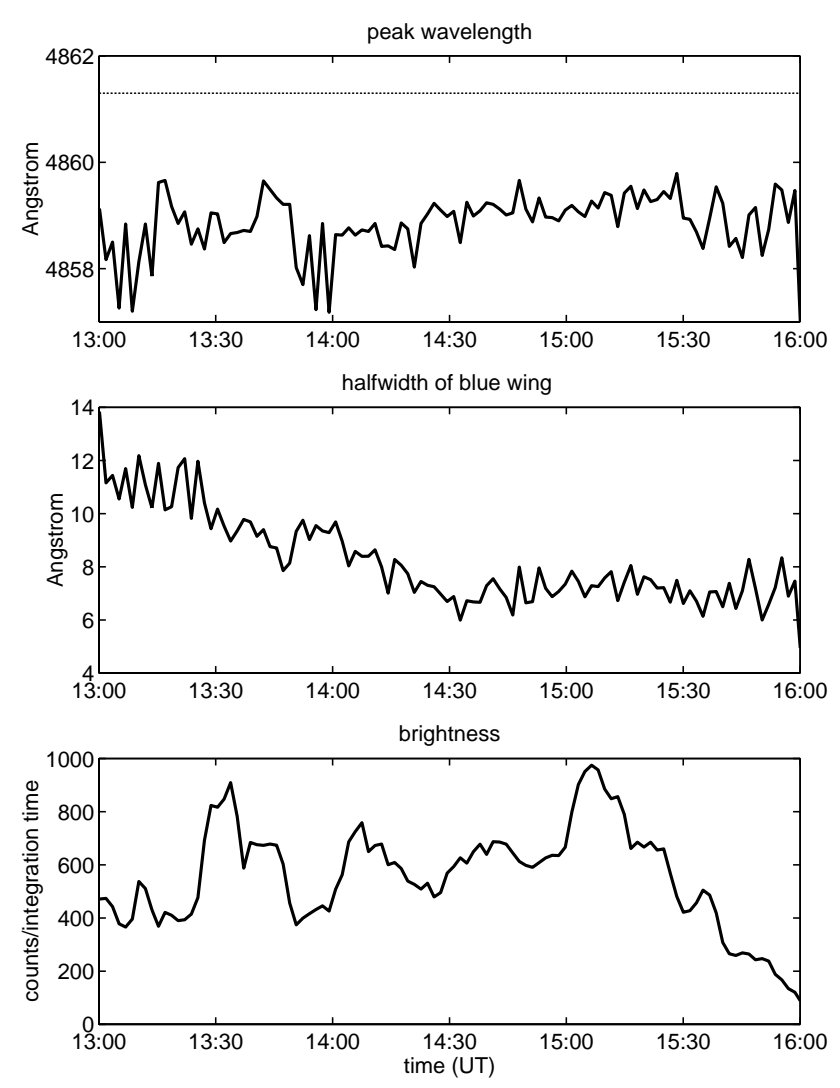

Fig. 14. Variations in fitted paramaters of profiles: (top) peak wavelength, (middle) width of blue wing and (bottom) intensity in counts per minute.

ment on Svalbard. The prolonged incidence of proton precipitation in an extended region encompassing Svalbard during the early afternoon hours gave the first opportunity to compare the changes in the measured hydrogen profiles with results of modeling, using as input several different energy spectra from satellites passing over Longyearbyen, and over the proton precipitation region. The changes in the profiles at $60 \mathrm{~s}$ resolution gave evidence for the variability of the incoming protons in flux and energy. After 13:00 UT, when the effect of solar scattering was reducing, several bursts in $\mathrm{H}_{\beta}$ emission were measured at Longyearbyen, as shown in Fig. 1 and Fig. 14, superimposed on a steady background of emission. As can be seen from the Lyman- $\alpha$ emissions from the IMAGE satellite in Fig. 1b, these formed a band which was centred over Longyearbyen at 13:00 UT but gradually moved south relative to the ground station, the result of the Earth's rotation. At 15:30 UT the effect of a southward turning of IMF $B_{z}$ caused a sudden expansion of the auroral oval, and consequently, a further movement of the peak emission southward of Longyearbyen. The changes in the measured $\mathrm{H}_{\beta}$ Doppler profiles appear to reflect this spatial change, with a decrease in the blue wing, and a small but steady decrease in the shift of the peak of the profiles, most probably indicating a reduction in mean energy of the incoming protons during the afternoon. 

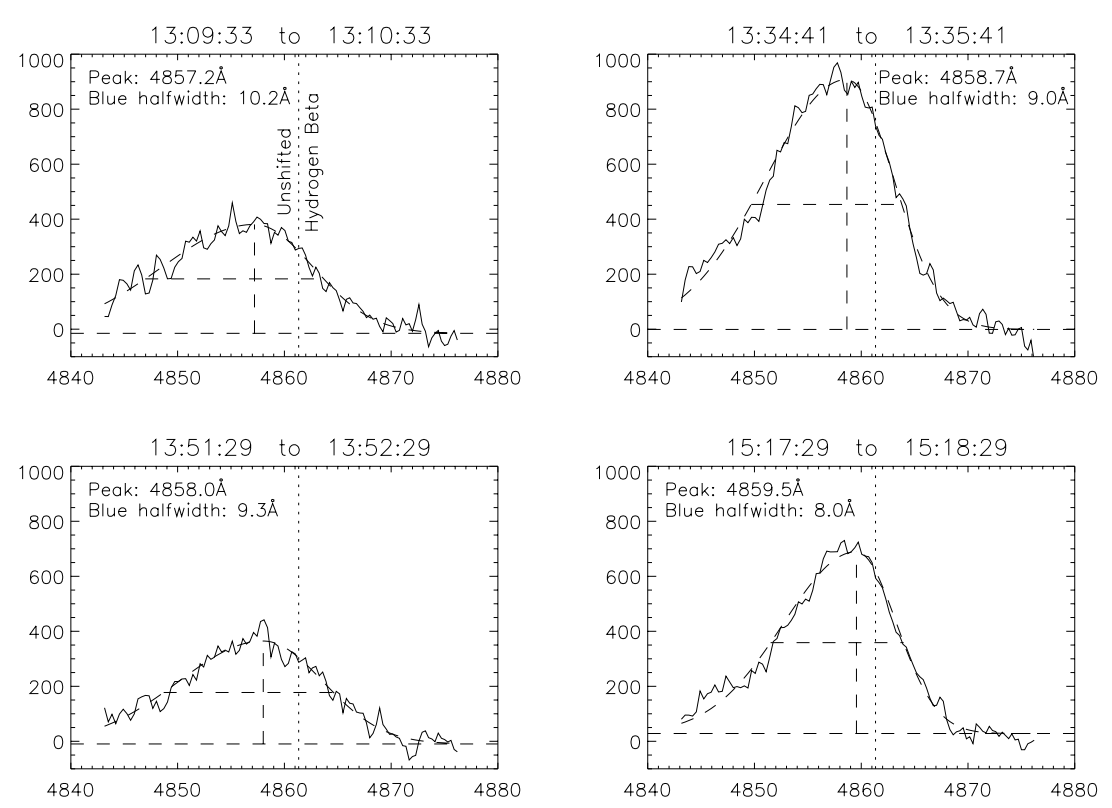

Fig. 15. Fitted profiles for four different times showing changes in the fitted peak and halfwidth of the blue wing. The intensity scale is in counts per minute.

In order to justify these conclusions a detailed comparison of measured and model profiles has been made. The best direct comparison is from FAST orbit 16928 at 15:28 UT. This is immediately before the time that the emissions moved south of the ground station and at the end of one of the bursts in flux. The satellite passed almost directly overhead at 15:28:30 UT. The model profiles and the integrated observed profiles (Fig. 13) are very close in shape for the peak value and most of the blue wing. The $60 \mathrm{~s}$ profile at 15:28 UT (Fig. 12) also compares well with the model result, even though the brightness was reduced at the end of the final burst of emission. The blue wing is more raised in the integrated profile, indicating higher mean energy of the incoming protons earlier in the interval. The raised blue wing reflects the presence of a high energy tail to the energy spectrum of the incoming protons. In order to provide an input spectrum to the model, we have estimated the contribution that this high energy tail makes to the spectrum, and it can be seen from the three model curves in Fig. 13 that changes could be made that would satisfy the measured shape. The contribution from a peak in energy of $\sim 5 \mathrm{keV}$ in the 15:31 UT spectrum is seen in the width of the profile near the peak value.

The measured red wing is mostly fitted well by the model results in the examples shown. In order to fit the red wing more precisely it may be necessary to take collisional angular redistribution up to higher energies in the model than has been done here. The effect of this change is seen in Fig. 16. Here the input spectrum from FAST at 15:28 UT has been applied to the model with collisional angular redistribution below $1 \mathrm{keV}$ and again below $50 \mathrm{keV}$, as an extreme example. If collisional angular redistribution is applied up to higher energies, particles of higher energies are scattered upwards. As a result, the red wing extends to larger wavelengths. The curves in Fig. 16 are normalised: the total brightness for the $50 \mathrm{keV}$ case is about $50 \%$ of the $1 \mathrm{keV}$ case, as in the former

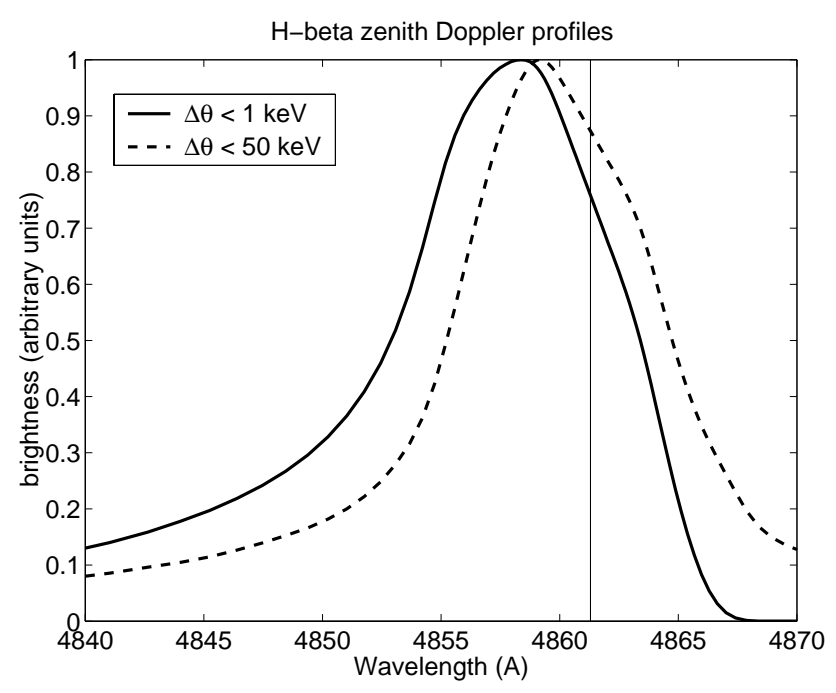

Fig. 16. Collisional angular redistribution applied below different energy thresholds.

fewer particles reach the E-region atmosphere and, therefore, less excitation occurs. The model has no restriction regarding the phase function and its dependence on energy. It has been assumed in the analysis that the phase function is constant over energy. Above $1 \mathrm{keV}$, the phase function is small. For this reason the results shown in Sect. 4 have collisional angular redistribution applied below $1 \mathrm{keV}$. This is an area that can be investigated with the acquisition of more data under different conditions.

It can also be seen from Fig. 16 that the effect of collisional angular redistribution at high energies is also a factor in producing a variation in the peak wavelength. Not only is the red wing more raised with the change in upper limit for the backscattered component, but the peak wavelength oc- 


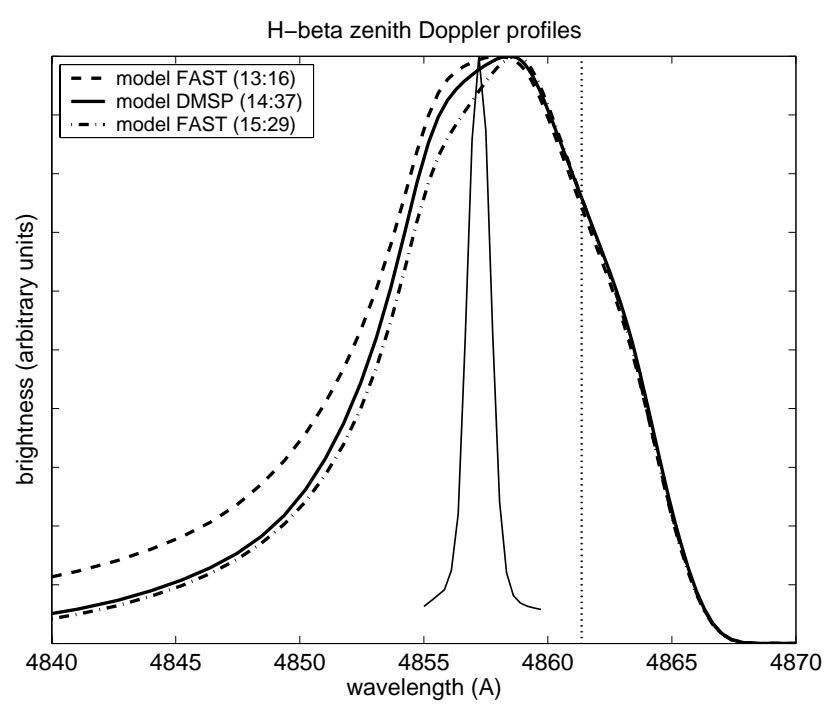

Fig. 17. Comparison of three different model runs corresponding to the best fit to measured profiles at times of satellite passes.

curs at longer wavelengths. The model results also indicate that a small change in the peak wavelength with a change in mean energy is predicted. Figure 17 shows three model profiles using satellite spectra from different times (13:16 UT, 14:37 UT and 15:29 UT), with different energy distributions. There is a small change in blue shift near the peak of the profiles, which matches quite well the measured results from these three times. More distinct changes could be achieved in the model results if anisotropy is taken into account.

The measured profiles show variations in the peak wavelength, with a move from shorter to longer wavelengths between 13:00 UT and 14:30 UT, although it can be seen from Fig. 14 and Fig. 15 that there are excursions from this trend, especially during the earlier part of the event. The observed trend agrees with the satellite particle measurements of the precipitating energy distributions, which show that there is a decrease in the mean energy between the two passes. The IMAGE Lyman- $\alpha$ data also show that temporal bursts in emission are superimposed on the background emission that is gradually moving southward away from Svalbard, indicating that the observed changes measured on the ground reflect the fact that the incoming protons have a changing energy distribution across the spatial extent of the region of precipitation. The energy spectrum with high energy tail occurs in the middle of the region. As the region of precipitation moves over the ground station the measured blue wing of the profiles reduces. The peak wavelength and the blue wing of the profiles are very closely linked; the shape of the whole profile gives the complete energy distribution of the incoming protons.

Although the comparison between measured and modeled profiles has been limited to times when spacecraft have passed close to Longyearbyen, there is still a problem of separating spatial and temporal changes measured on the ground. For the 15:28 UT pass, the location of Longyearbyen is very close to the edge of a separate emission structure, and this makes the interpretation of the ground-based measurements more crucial to the understanding of the changes in energy input. It shows that temporal and spatial changes of precipitation on quite short time scales are the likely explanation for the very variable $\mathrm{H}_{\beta}$ line profiles that are measured. At the time of the DMSP pass at 14:37 UT, it appears from the Lyman- $\alpha$ images from IMAGE that the F12 satellite skirted the edge of the region of most intense proton emission. For the 13:16 UT FAST pass the IMAGE data (Fig. 1b) show that Longyearbyen is close to the middle of the precipitation region at this time, and therefore an input spectrum from the middle of the intense region is more likely to be appropriate. However, the spectra measured by FAST do not extend high enough in energy to make this a valid comparison here. Again, there is much variation in the energy spectra measured by FAST, which is interpreted as spatial changes. These could indeed be related to temporal bursts of emission, and varying profile shapes measured on the ground during this interval, seen in Fig. 1 and Fig. 14. Another limitation of this comparison between measured and modeled profiles is the fact that the model is one-dimensional. Therefore, the effect of horizontal transport is not included. Proton beams are spread out when they are partially neutralised through collisions (Davidson, 1965; Johnstone, 1972; Iglesias and Vondrak, 1974; Kozelov, 1993). There could be a small contribution to the measured profiles from neighbouring regions, which is not accounted for in the model. The spatial variations measured by FAST are of the order of $20 \mathrm{~km}$.

The combination of global images and satellite particle measurements is particularly powerful in choosing the input spectra most suited to model the resulting emission profiles measured from the ground. The results discussed here are the first such comparison of measured $\mathrm{H}_{\beta}$ profiles at high resolution with model profiles obtained from independent satellite measurements of the incoming energy distributions.

\section{Conclusions}

1. Measured $\mathrm{H}_{\beta}$ profiles give an excellent estimate of the variations of incoming proton mean energy and energy flux. During a strong proton precipitation event over Svalbard, mean energies of a few $\mathrm{keV}$ to $>15 \mathrm{keV}$ were measured by particle detectors on satellites flying over Longyearbyen, which corresponded to changes in the shape of Doppler profiles measured with a groundbased imaging spectrograph (HiTIES). The high resolution and sensitivity of the HiTIES instrument allowed for small changes in the peak wavelength to be detected. These changes were most probably the result of changes in the energy spectra of the incident particles. Further investigations of the source of these changes will need to consider the anisotropy of the incident proton beam and the dependence in energy of the phase function.

2. Modelled $\mathrm{H}_{\beta}$ profiles, using the measured energy spectra from satellites as input, closely resemble the mea- 
sured profiles in shape. In particular, the reduction in the extent of the blue wing reflects the decreasing mean energy during the event. As well, the presence of an extended red wing confirms the result of Lummerzheim and Galand (2001) that there is a backscattered component of hydrogen atoms. This is the first observation on the dayside of a red wing of physical origin.

3. Lyman- $\alpha$ measurements from IMAGE/SI12 show how the large-scale emission region over Svalbard is related to the measurements on the ground. In this event we interpret changes in the peak wavelength and profile shape measured with HiTIES as the result of both spatial and temporal changes in the precipitating flux of protons over Svalbard. The global images are invaluable in the choice of the most appropriate particle data from FAST and DMSP satellites to give realistic input spectra for the model.

4. The high energy part of the input spectra is not measured by satellite detectors above $30 \mathrm{keV}$. It is clear from the measured $\mathrm{H}_{\beta}$ profiles presented here that a large proportion of the incoming protons have energies above this value. The measured emission profile could provide even more information about the high energy tail of the incoming proton distribution with a proposed change of filter to include more of the blue shifted wing below $4843 \AA$. The HiTIES instrument thus provides a continuous measure of the precipitating energy distribution of protons over Svalbard, dark and cloud-free skies permitting.

\section{Appendix The Galand model of the hydrogen Doppler profile}

The present model solves the coupled transport equations for the proton and hydrogen fluxes $\Phi_{P}(s, E, \mu)$ and $\Phi_{H}(s, E, \mu)$ as a function of the distance $s$ along the magnetic field line, energy $E$, and cosine of the pitch-angle $\mu$. The equations are (Galand, 1996; Galand et al., 1997; Galand and Richmond, 1999):

$$
\begin{aligned}
& \mu \frac{\partial}{\partial s} \Phi_{P}(s, E, \mu)+\mu \frac{d \mu}{d s} \cdot \frac{\partial}{\partial \mu} \Phi_{P}(s, E, \mu) \\
& -\sum_{\alpha} n_{\alpha}(s) \sum_{k} \int d \mu^{\prime} \frac{\partial}{\partial E}\left(L_{\alpha, P}^{k}\left(E, \mu^{\prime} \rightarrow \mu\right) \cdot \Phi_{P}\left(s, E, \mu^{\prime}\right)\right) \\
& -\sum_{\alpha} n_{\alpha}(s) \int d \mu^{\prime} \frac{\partial}{\partial E}\left(L_{\alpha}^{01}\left(E, \mu^{\prime} \rightarrow \mu\right) \cdot \Phi_{H}\left(s, E, \mu^{\prime}\right)\right) \\
& =-\sum_{\alpha} n_{\alpha}(s) \sigma_{\alpha, P}^{T}(E) \Phi_{P}(s, E, \mu)+\sum_{\alpha} n_{\alpha}(s) \\
& \sum_{k} \int d \mu^{\prime} \zeta_{\alpha, P}^{k}\left(E, \mu^{\prime} \rightarrow \mu\right) \cdot \sigma_{\alpha, P}^{k}(E) \cdot \Phi_{P}\left(s, E, \mu^{\prime}\right) \\
& +\sum_{\alpha} n_{\alpha}(s) \int d \mu^{\prime} \zeta_{\alpha}^{01}\left(E, \mu^{\prime} \rightarrow \mu\right) \\
& \cdot \sigma_{\alpha}^{01}(E) \cdot \Phi_{H}\left(s, E, \mu^{\prime}\right)
\end{aligned}
$$

and

$$
\begin{aligned}
& \mu \frac{\partial}{\partial s} \Phi_{H}(s, E, \mu)+\mu \frac{d \mu}{d s} \cdot \frac{\partial}{\partial \mu} \Phi_{H}(s, E, \mu)-\sum_{\alpha} n_{\alpha}(s) \\
& \sum_{k} \int d \mu^{\prime} \frac{\partial}{\partial E}\left(L_{\alpha, H}^{k}\left(E, \mu^{\prime} \rightarrow \mu\right) \cdot \Phi_{H}\left(s, E, \mu^{\prime}\right)\right) \\
& -\sum_{\alpha} n_{\alpha}(s) \int d \mu^{\prime} \frac{\partial}{\partial E}\left(L_{\alpha}^{10}\left(E, \mu^{\prime} \rightarrow \mu\right) \cdot \Phi_{P}\left(s, E, \mu^{\prime}\right)\right) \\
& =-\sum_{\alpha} n_{\alpha}(s) \sigma_{\alpha, H}^{T}(E) \cdot \Phi_{H}(s, E, \mu)+\sum_{\alpha} n_{\alpha}(s) \\
& \sum_{k} \int d \mu^{\prime} \zeta_{\alpha, H}^{k}\left(E, \mu^{\prime} \rightarrow \mu\right) \cdot \sigma_{\alpha, H}^{k}(E) \cdot \Phi_{H}\left(s, E, \mu^{\prime}\right) \\
& +\sum_{\alpha} n_{\alpha}(s) \int d \mu^{\prime} \zeta_{\alpha}^{10}\left(E, \mu^{\prime} \rightarrow \mu\right) \cdot \sigma_{\alpha}^{10}(E) \cdot \Phi_{P}\left(s, E, \mu^{\prime}\right) .(\mathrm{A} 2)
\end{aligned}
$$

In both equations the first term on the left side specifies the change in particle flux along a magnetic field line. The second term accounts for the variation in pitch-angle in a non-uniform magnetic field. The third and fourth terms represent the energy degradation through non-charge-changing reactions (excitation, ionisation and elastic scattering) and charge-changing reactions (stripping (01) and capture (10)), respectively. The energy degradation is taken into account through a dissipative force.

On the right side of the equations the collision term is reduced to pure elastic reactions for all processes. The energy losses are included on the left-hand side. The first term accounts for particle loss by all collision processes. The second term describes particle gain through angular scattering while the last term gives the particle gain through angular redistribution in the charge-changing reactions.

Denoting protons and hydrogen atoms by $\gamma(=P, H)$, collisions with neutral species by $\alpha\left(=\mathrm{N}_{2}, \mathrm{O}_{2}, \mathrm{O}\right)$ and collision type by $k$ ( = excitation, ionisation, elastic scattering) the energy loss function is

$$
\begin{aligned}
& L_{\alpha, \gamma}^{k}\left(E, \mu^{\prime} \rightarrow \mu\right)= \\
& W_{\alpha, \gamma}^{k}\left(E, \mu^{\prime} \rightarrow \mu\right) \sigma_{\alpha, \gamma}^{k}(E) \zeta_{\alpha, \gamma}^{k}\left(E, \mu^{\prime} \rightarrow \mu\right),
\end{aligned}
$$

where $W_{\alpha, \gamma}^{k}$ is the energy loss, $\sigma_{\alpha, \gamma}^{k}$ is the collision cross section. $\zeta_{\alpha, \gamma}^{k}$ is the normalized phase function which specifies the angular redistribution. Applicability of the continuous energy loss approximation is discussed and justified by Galand (1996) who also gives numerical values for the $W \mathrm{~s}$ and $\sigma \mathrm{s}$. The phase function uses the screened Rutherford cross section. Denoting the scattering angle by $\Theta$,

$\zeta(\cos \Theta)=\frac{4 \varepsilon(1+\varepsilon)}{(1+2 \varepsilon-\cos \Theta)^{2}}$,

where

$\cos \Theta=\mu \mu^{\prime}+\sqrt{1-\mu^{\prime 2}} \sqrt{1-\mu^{2}} \cos \left(\phi-\phi^{\prime}\right)$,

and $\mu$ and $\mu^{\prime}$ are the cosine of the pitch-angle before and after collision; $\phi$ and $\phi^{\prime}$ are azimuthal angles before and after collisions, respectively, $\varepsilon$ is an energy dependent screening 
parameter that increases with decreasing energy for electron collisions. The variation of $\varepsilon$ for protons and hydrogen atoms is not known, and a constant value of $1 \times 10^{-3}$, is adopted below $1 \mathrm{keV}$ (Galand et al., 1998). Forward scattering approximation is applied above $1 \mathrm{keV}$, unless specified otherwise.

$L_{\alpha}^{01}$ in the proton transport equation $(\mathrm{P})$ is the loss function for stripping of a hydrogen atom, which becomes a source of protons while $L_{\alpha}^{10}$ is the loss function for electron capture which becomes a source of hydrogen atoms in the hydrogen transport equation $(\mathrm{H})$. The corresponding phase functions are $\zeta_{\alpha}^{01}$ and $\zeta_{\alpha}^{10}$. Terms in the transport equations that include these parameters represent the coupling between proton and hydrogen atom fluxes.

The total absorption cross section, $\sigma_{\alpha, \gamma}^{T}(E)$ is the sum of the ionization, excitation, elastic and charge-changing cross sections. $n_{\alpha}(s)$ is the number density of neutral particles $\alpha$ at distance $s$ along the field line. The MSIS 90 (Hedin, 1991) neutral atmosphere is adopted, being a function of location, local time, magnetic activity and solar index.

Compared with collisional angular redistribution the effect of the magnetic mirror force can be neglected when simulating the Doppler profile of hydrogen. The effect of magnetic mirroring is mainly seen above $250 \mathrm{~km}$, whereas most of the excitation occurs at altitudes below this. The second term on the left side of the $\mathrm{P}$ and $\mathrm{H}$ transport equations is set to zero in the present application.

Proton precipitation is sufficiently uniform over extended regions to justify a one-dimensional transport simulation in a plane parallel atmosphere (Davidson, 1965). A proton flux of specified energy and pitch-angle distributions precipitates at the upper boundary, $500 \mathrm{~km}$. In the energy range of auroral proton fluxes absorption is complete at the lower boundary, $95 \mathrm{~km}$.

The line-of-sight integrated Doppler profile of an emission line in the magnetic zenith is

$D(\lambda)=\iint d s d v_{\perp} P_{r \ell v}\left(s, \lambda, v_{\perp}\right)$,

with photons $\mathrm{cm}^{-2} \mathrm{~s}^{-1} \mathrm{~nm}^{-1}$ expressed in terms of the volume emission rate $P_{r l v}\left(s, \lambda, v_{\perp}\right)$ integrated over the distance $s$ and the velocity component $v_{\perp}$ perpendicular to the magnetic field. In terms of the variables $s, E, \mu$ and applied to the $\mathrm{H}_{\beta}$ line (Galand, 1996; Galand et al., 1998),

$$
\begin{aligned}
& P_{r \ell v}\left(s, \lambda, v_{\perp}\right)= \\
& \frac{m c}{\lambda_{o}}\left(1-\frac{v_{\|}}{c}\right)^{2} \frac{v_{\perp}}{\sqrt{v_{\|}^{2}+v_{\perp}^{2}}} \cdot P_{r H_{\beta}}(s, E, \mu),
\end{aligned}
$$

where $\lambda_{0}$ is the unshifted wavelength, $m$ the mass of the hydrogen atom; $c$ is the speed of light; $v_{\|}$is the velocity component along the magnetic field line

$$
\begin{aligned}
& v_{\|}=\mu \sqrt{\frac{2 E}{m}} ; \quad v_{\perp}=\sqrt{1-\mu^{2}} \sqrt{\frac{2 E}{m}} ; \\
& \lambda=\frac{\lambda_{o}}{1-\frac{\mu}{c} \sqrt{\frac{2 E}{m}}} .
\end{aligned}
$$

The production rate of $\mathrm{H}_{\beta}$ photons is

$P_{r H_{\beta}}(s, E, \mu)=$
$2 \pi \sum_{\alpha} \sum_{\gamma} n_{\alpha}(s) \sigma_{\alpha, \gamma}^{H_{\beta}}(E) \Phi_{\gamma}(s, E, \mu)$,

where the emission cross section as a function of energy, $\sigma_{\alpha, \gamma}^{H_{\beta}}$, is taken from Van Zyl and Newman (1980) and from Yousif et al. (1986) for collisions of $\mathrm{P}$ and $\mathrm{H}$ with $\mathrm{N}_{2}$ and $\mathrm{O}_{2}$. The emission cross section for collisions with $\mathrm{O}$ is assumed to be 0.7 of the $\mathrm{O}_{2}$ cross section (Strickland et al., 1993).

Acknowledgements. The DMSP particle detectors were designed by Dave Hardy of AFRL, and data obtained from JHU/APL. The authors thank Dave Hardy, Fred Rich and Patrick Newell for its use, and in particular Fred Rich for providing data specific to our needs. The authors thank C. S. Deehr for the Meridian Scanning Photometer data. ACE and Wind data were obtained from CDAWeb and we acknowledge the following PIs: D. J. McComas, R. Lepping and K. Ogilvie. Thanks are offered to Steve Morley and Katie Throp for help with data reduction. B.S.L. was supported by a grant from the PPARC in the UK which also funded the spectrograph. M.G. was supported by NSF grant ATM-0003175, D.L. and M.H.R. by NASA grants NAG5-11694 and NAG5-12192, L.M.P. by NASA grant NAG5-3596 and H.U.F. by NASA grant NAS5-96020.

Topical Editor U. P. Hoppe thanks two referees for their work in evaluating this paper.

\section{References}

Basu, B., Jasperse, J. R., Strickland D. J., and Daniell R. E.: Transport theoretic model for the electron-proton-hydrogen atom aurora, 1, Theory, J. Geophys. Res. 98, 21 517-21 532, 1993.

Baumgardner, J., Flynn, B. and Mendillo, M.: Monochromatic imaging instrumentation for applications in aeronomy of the earth and planets, Optical Eng., 32, 3028-3032, 1993.

Chakrabarti, S., Pallamraju, D., Baumgardner, J., and Vaillancourt, J.: HiTIES: A High Throughput Imaging Echelle Spectrograph for ground-based visible airglow and auroral studies, J. Geophys. Res., 106, 30 337-30 348, 2001.

Chamberlain, J. W.: Physics of the Aurora and Airglow, Academic Press, New York, 1961.

Davidson, G. T.: Expected spatial distribution of low-energy protons precipitated in the auroral zones, J. Geophys. Res., 70, 1061, 1965.

Deehr, C. S., Lorentzen, D. A., Sigernes, F., and Smith, R. W.: Dayside auroral hydrogen emission as an aeronomic signature of magnetospheric boundary layer processes, Geophys. Res. Lett., 25, 2111-2114, 1998.

Degen, V.: Modeling of $\mathrm{N}_{2}^{+}$First Negative spectra excited by electron impact, J. Quant. Spectroscopic Radiat. Transfer, 18, 113119, 1977.

Eather, R. H.: Auroral proton precipitation and hydrogen emissions, Rev. Geophys., 5, 207-285, 1967.

Eather, R. H. and Burrows, K, M.: Excitation and ionisation by auroral protons, Austral. J. Phys., 19, 309, 1966.

Eather, R. H. and Jacka, F.: Auroral hydrogen emission, Australian J. Phys., 19, 241-274, 1966.

Frey, H. U., Mende, S. B., Carlson, C. W., Gerard, J.-C., Hubert, B., Spann, J., Gladstone, R., and Immel, T. J.: The electron and 
proton aurora as seen by IMAGE-FUV and Fast, Geophys. Res. Lett., 28, 1135-1138, 2001.

Galand, M.: Transport des protons dans l'ionosphère aurorale, Thèse de 3ème cycle, Spécialité Astrophysique et Milieux Dilués, Univ. Joseph Fourier, Grenoble, France, 1996.

Galand, M., Lilenstein, J., Kofman, W., and Sidje, R. B.: Proton transport model in the ionosphere: 1 Multistream approach of the transport equations, J. Geophys. Res., 102, 22 261-22 272, 1997.

Galand, M., Lilenstein, J., Kofman, W., and Lummerzheim, D.: Proton transport model in the ionosphere: 2, Influence of magnetic mirroring ad collisions on the angular redistribution in a proton beam, Ann. Geophysicae, 16, 1308-1321, 1998.

Galand, M. and Richmond, A. D.: Magnetic mirroring in an incident proton beam J. Geophys. Res., 104, 4447-4456, 1999.

Galand, M. and Richmond, A. D.: Ionospheric electrical conductances produced by auroral proton precipitation J. Geophys. Res., 106, 117-125, 2001

Gartlein, C. W.: Auroral spectra showing broad hydrogen lines, Trans. Ameri. Geophys. Union, 31, 18-20, 1950.

Gartlein, C. W.: Protons and the aurora, Phys. Rev., 81, 463-464, 1951.

Gérard, J.-C., Hubert, B., Bisikalo, D. V., and Shematovich, V. I.: A model of the Lyman-alpha line profile in the proton aurora, $\mathrm{J}$. Geophys Res., 105, 15 795-15 806, 2000.

Harang, O. and Pettersen, H.: Variation in width of the $\mathrm{H}$ alpha line in aurora, Planetary Space Sci., 15, 1599, 1967.

Hedin, A. E.: Extension of the MSIS thermosphere model into the middle and lower atmosphere, J. Geophys Res., 96, 1159-1172, 1991.

Henriksen, K., Fedorova, N. I., Totunova, G. F., Deehr, C. S, Romick, G. J., and Sivjee, G. G.: Hydrogen emissions in the polar cleft, J. Atmospheric Terr. Phys., 47, 1051-1056, 1985.

Hunten, D. M.: Some photometric observations of auroral spectra, J. Atmospheric Terr. Phys., 7, 141-151, 1955.

Iglesias, G. E. and Vondrak, R. R.: Atmospheric spreading of protons in auroral arcs,J. Geophys. Res., 79, 280, 1974.

Johansen, O. E. and Omholt, A.: Variations in the Doppler profile of Halpha in aurorae, Planetary Space Sci., 11, 1223-1231, 1963.

Johnstone, A. D.: The spreading of a proton beam by the atmosphere, Planet. Space Sci., 20, 292, 1972.

Kerr, R. B. and Hecht, J. H.: Measurement of geocoronal Balmer alpha in the auroral zone, J. Geophys. Res., 101, 189-194, 1996.

Kozelov, B. V.: Influence of the dipolar magnetic field on transport of proton-H atom fluxes in the atmosphere, Ann. Geophysicae,11, 697-704, 1993.

Lanchester, B. S., Rees, M. H. Robertson, S. C., Galand, M., Lummerzheim, D., Baumgardner, J., Mendillo, M., Furniss, I., and Aylward, A. D.: Proton and electron precipitation over Svalbard - first results from a new Imaging Spectrograph (HiTIES), Proc. of Atmospheric Studies by Optical Methods, SGO Pubs 92, 3336, 2003.

Lockwood, M., Lanchester, B. S., Frey, H., Throp, K., Morley, S., Milan, S. E., and Lester, M.: IMF Control of Cusp Proton Emission Intensity and Dayside Convection: implications for component and anti-parallel reconnection, Ann. Geophysicae, 21,
955-982, 2003.

Lorentzen, D. A., Sigernes, F., and Deehr, C. S.: Modeling and observations of dayside auroral hydrogen emission Doppler profiles, J. Geophys. Res., 103, 17 479-17 488, 1998.

Lummerzheim, D. and Galand, M.: The profile of the hydrogen $\mathrm{H}_{\beta}$ emission line in proton aurora, J. Geophys. Res., 106, 23-31, 2001.

McWhirter, I., Furniss, I., Aylward, A. D., Lanchester, B. S., Rees, M. H., Robertson, S. C., Baumgardner, J., and Mendillo, M.: A new spectrograph platform for auroral studies in Svalbard, Proc. of Atmospheric Studies by Optical Methods, SGO Pubs 92, 7376, 2003.

Meinel, A. B.: Doppler-shifted auroral hydrogen emission, Astrophys. J., 113, 50-54, 1951

Montalbetti, R.: Photoelectric measurements of hydrogen emissions in aurora and airglow, J. Atmospheric Terr. Phys. 14, 200212, 1959

Omholt, A.: Photometric observations of rayed and pulsating aurorae, Astrophys. J., 126, 461-463, 1957.

Rees, M. H.: On the interaction of auroral protons with the Earth's atmosphere, Planet. Space Sci., 30, 463-472, 1982.

Sigernes, F., Lorentzen, D. A., Deehr, C. S., and Henriksen, K.: Calculation of auroral Balmer volume emission height profiles in the upper atmosphere, J. Atmospheric Terr. Phys., 56, 503-508, 1994.

Sigernes, F.: Estimation of initial auroral proton energy fluxes from Doppler profiles, J. Atmospheric Terr. Phys., 58, 1871-1883, 1996.

Solomon, S. C.: Auroral particle transport using Monte Carlo and hybrid methods, J. Geophys. Res., 106, 107-116, 2001.

Söraas, F., Lindalen, H. R., Måseide, K., Egeland, A., Sten, T. A., and Evans, D. S.: Proton precipitation and the $\mathrm{H}_{\beta}$ emission in a postbreakup auroral glow, J. Geophys. Res., 79, 1851-1859, 1974.

Söraas, F., Måseide, K., Torheim, P., and Aarnes, K.: The Dopplershifted auroral Hbeta emission: a comparison between observations and calculations, Ann. Geophysicae, 12, 1052-1064, 1994

Strickland, D. J., Daniell, Jr., R. E., Jasperse, J. R., and Basu, B.: Transport-theoretic model for the electron-proton-hydrogen atom aurora, 2, Model results, J. Geophys. Res., 98, 21,533$21548,1993$.

Synnes, S. A., Söraas, F., and Hansen, J. P.: Monte-Carlo simulation of proton aurora, J. Atmos. Sol.-Terr. Phys., 60, 1695, 1998.

Van Zyl, B. and Neumann, H.: H-alpha and H-beta emission cross sections for low energy $\mathrm{H}$ and $\mathrm{H}^{+}$collisions with $\mathrm{N}_{2}$ and $\mathrm{O}_{2}, \mathrm{~J}$. Geophys. Res., 85, 6006-6010, 1980.

Vegard, L.: Hydrogen showers in the auroral region, Nature, 144, 1089-1090, 1939.

Vegard, L.: Emission spectra of night sky and aurora, Reports of the Gassiot Committee, The Physical Society of London, 82, 1948.

Yousif, F. B., Geddes, J., and Gilbody, H. B.: Balmer alpha emission in collisions of $\mathrm{H}, \mathrm{H}^{+}, \mathrm{H}_{2}^{+}$with $\mathrm{N}_{2}, \mathrm{O}_{2}$, and $\mathrm{H}_{2} \mathrm{O}$, J. Phys. B.,, 19, 217-231, 1986.

Zwick, H. H. and Shepherd, G. G.: Some observations of hydrogen line profiles in the aurora, J. Atmospheric Terr. Phys., 25, 604$607,1963$. 